\title{
Overview of Childhood Cancers at a Regional Cancer Centre in North-East India
}

\author{
Munlima Hazarika ${ }^{1 *}$, Manigreeva Krishnatreya ${ }^{2}$, Cidananda Bhuyan ${ }^{1}$, Bhargab \\ Jyoti Saikia ${ }^{1}$, Amal Chandra Kataki ${ }^{3}$, Pintu Nandy ${ }^{2}$, Monalisha Hazarika ${ }^{1}$, Partha \\ Sarathi Roy ${ }^{1}$
}

\begin{abstract}
Childhood cancers are relatively uncommon in comparison to adult cancers. There is no literature available to shed light on clinic-pathological types and patterns of care for childhood cancers in our population in North-East India. In this analysis we therefore tried to determine the common childhood cancers diagnosed in our institute, clinical profile of the patients, types of treatment and compliance, and median survival estimates. Leukemia was most common, followed by retinoblastoma, central nervous system tumours and lymphomas. Ascertaining the clinic-pathological profile of childhood cancers in our population is essential for allocation and management of resources for this small but important group of patients.
\end{abstract}

Keywords: Childhood cancer - pattern treatment types - North East India

Asian Pac J Cancer Prev, 15 (18), 7817-7819

\section{Introduction}

Pediatric cancer is rare and biologically it is not similar to cancer in the adults. An estimated 160,000 new cases of children below 15 years of age are diagnosed with cancer each year worldwide, with 90,000 deaths attributed to cancer (Ferlay et al., 2004). In India approximately 45,000 children are diagnosed with cancer every year (Arora et al., 2009). The proportion of childhood cancers (0-14) relative to all cancers reported by Indian cancer registries varied from $0.8 \%$ to $5.8 \%$ in boys, and from $0.5 \%$ to $3.4 \%$ in girls (National Cancer Registry Programme, 2013).

In the developed countries, the survival of childhood cancers is better than developing countries (Ferlay et al., 2010; Wiangnon et al., 2011; Satyanarayana et al., 2014). Also, in low and middle income countries $80 \%$ of world's children live but, $56 \%$ of the cases and $64 \%$ of the deaths occur each year due to limited access to curative treatment including the lack of availability of common chemotherapeutic agents, cost of treatment, late stage at presentation, and limited radiotherapy and surgical resources (Ferlay et al., 2010; Satyanarayana et al., 2014). In addition, even when adequate oncologic treatments are available, disparities in education and socioeconomic conditions, coupled with inefficient or suboptimal health care delivery, result in poor outcomes for children diagnosed with cancer in low and middle income countries (Slone et al., 2014). Considerable inter-regional variation in the incidence and mortality of childhood cancer in India needs to be ascertained (Arora et al., 2009). In North- East
India, the age adjusted incidences of most of adult cancers are very high; however, there are no published data on childhood cancers.

In this study we therefore tried to determine the common childhood cancers diagnosed in our population, clinical profile of the patients, types of treatment, compliance to treatment and median survival estimates.

\section{Materials and Methods}

This study was commenced after obtaining permission from institutional scientific committee. This retrospective study was carried out at a regional cancer center in the North East India. A separate registry is maintained in the department of pediatric oncology at our institute. All cases of childhood cancer from 0-14 years of age that was registered from 1st April 2013 to $31^{\text {st }}$ March 2014 were included in this study. The case records were analyzed to show the descriptive profile of the patients. All cases were histopathologically or cytologically confirmed cases of malignancy. All the patients also underwent all diagnostic and metastatic workup. On the basis of medical record review, treatment compliance for each patient was categorized as (1) completed or undergoing treatment; (2) refused treatment and (3) abandoned treatment. Abandonment of treatment was defined as the termination of care by the parent/caregiver and/or not presenting for scheduled treatment for four weeks from the scheduled date of treatment at the time of data record. If a child returned for treatment after 4 weeks, his/her classification 
Munlima Hazarika et al

remained unchanged. Refusal of treatment was defined as no initiation of treatment after the diagnosis of cancer was made. The follow-up for survival was an active one. The survival was estimated from the date of first diagnosis.

\section{Statistical analyses}

The results are presented as descriptive statistics. The median survival was calculated using Statistical Package for Social Sciences.

\section{Results}

\section{Patient profile}

A total of 145 children with cancers were registered. Of the participants included in the analyses, $60 \%$ (87/145) were boys and $40 \%$ (58/145) were girls. Highest number of patients was observed in the age group of 5-9years $(34.5 \%)$ as shown in Table 1.

\section{Types of cancer}

The five most common diagnoses were leukemia $26.9 \%(39 / 145)$, retinoblastoma was seen in $15.9 \%$ $(23 / 145)$, central nervous system (CNS) tumors in $14.5 \%$ $(21 / 145)$, lymphoma in $13.1 \%(19 / 145)$ children, bone tumors in $9.7 \%(14 / 145)$ and germ cell tumor in $9.7 \%$ (7/145) children (Table 2 ). The most common childhood malignancy was retinoblastoma $(43.7 \%, 21 / 48)$ in $<5$ years age group followed by leukemia $(27.1 \%, 13 / 48)$, and in 5-9 years and 10-14 years leukemia was seen in $30 \%(15 / 50)$ and $23.4 \%(11 / 47)$ patients respectively. This was followed by lymphoma $(28 \%, 14 / 50)$ in $5-9$ years and CNS tumors $(19.1 \%, 9 / 47)$ in $10-14$ years. The common histological sub types were acute lymphoid leukemia (ALL) was seen in 19.3\% (28/145), Hodgkin's lymphoma in $8.2 \%(12 / 145)$, acute myeloid leukemia in $6.8 \%(10 / 158)$, Ewing's sarcoma in $6.2 \%(9 / 145)$, non Hodgkin's lymphoma in $4.8 \%$ (7/145), and astrocytoma in $4.1 \%(6 / 145)$ patients (Table 2).

\section{Treatments planned and compliance}

In our observation $65.5 \%$ of the patients received chemotherapy (CT) and $40.7 \%$ received radiotherapy (RT) in combination with other modalities. Out of all patients who had received RT, $13.8 \%$ received concomitant chemo- radiotherapy (CT+RT). 26.2\% underwent surgery in combination with RT and/or CT. $81.4 \%$ of the patients completed a treatment regimen or were actively undergoing treatment. $11.7 \%$ refused treatment after diagnosis was confirmed and 5.5\% abandoned after

Table 1. It Shows the Gender and Age Group Distribution of Childhood Cancers Registered at Our Institute

\begin{tabular}{llll}
\hline Patient's profile & & Number & $\%$ \\
\hline & Gender & & \\
& Male & 87 & 60 \\
& Female & 58 & 40 \\
Age group & $0-4$ & 48 & 33.1 \\
& $5-9$ & 50 & 34.5 \\
& $10-14$ & 47 & 32.4 \\
\hline
\end{tabular}

$* \%=$ percentage
Table 2. Various Types of Childhood Cancers in Our Population

\begin{tabular}{lcr}
\hline Types of Pediatric cancer & Frequency & $\%$ \\
\hline Leukemia & 39 & 26.9 \\
Retinoblastoma & 23 & 15.9 \\
CNS Tm & 21 & 14.5 \\
Lymphoma & 19 & 13.1 \\
Bone Tm & 14 & 9.7 \\
Germ Cell Tm & 7 & 4.8 \\
Kidney Tm & 5 & 3.4 \\
Nasopharynx & 3 & 2.1 \\
Others & 14 & 9.6 \\
Leading histological sub types & & \\
ALL & 28 & 19.3 \\
Hodgkin's lymphoma & 12 & 8.2 \\
AML & 10 & 6.8 \\
Ewing's sarcoma & 9 & 6.2 \\
NHL & 7 & 4.8 \\
Astrocytoma & 6 & 4.1 \\
\hline *CNS=central nervous system, Tm=tumor & &
\end{tabular}

Table 3. Types of Treatment Instituted and Compliance to Treatment in Childhood Cancers in our Population

\begin{tabular}{lrr}
\hline & Frequency & Percent \\
\hline CT & 60 & 41.4 \\
RT & 9 & 6.2 \\
Surgery & 1 & 0.7 \\
CT+RT & 20 & 13.8 \\
S+RT & 22 & 15.2 \\
S+CT+RT & 8 & 5.5 \\
Palliative & 1 & 0.7 \\
S+CT & 7 & 4.8 \\
Treatment compliance & & \\
Treated or undergoing treatment & 118 & 81.4 \\
Abandonment & 8 & 5.5 \\
Refused & 17 & 11.7 \\
Palliative & 2 & 1.4 \\
*CT=chemotherapy, RT=radiotherapy, S=surgery &
\end{tabular}

initiation of treatment (Table 3).

Median survival

In the present analysis the information on death was obtained in 42 patients and rests were lost to follow-up. The median survival in this short follow-up period was 57 days (standard error $[\mathrm{SE}]=15.6$ ) and the median survival in $<5$ years was 24 days, in the 5-9 years it was 54 days and in 10-14 years it was 135 days (Log Rank (MantelCox $)$, Chi square $=4.464, \mathrm{P}=0.107)$.

\section{Discussion}

In India presently Population-based cancer registries (PBCR) under the National Cancer Registry Programme have been running actively. However, PBCRs have inherent problems, the primary one being the difficulty of ensuring comprehensive and complete data collection for ascertaining the exact age adjusted incidence of childhood cancers of our population. There are further problems in India, as cancer is not a notifiable disease (Arora et al., 2009). Due to non availability of proper pediatric cancer registry or pediatric oncology database, there is a lack of insight into the profile of pediatric cancer cases from 
this part of the country. We had identified the disease characteristics of pediatric cancer patients representing our population. In comparison of childhood cancers amongst different hospital based registries of India, it showed the lowest relative proportion of $1.7 \%$ at our hospital cancer registry (National Cancer Registry Programme., 2013). In our analysis boys were 1.5 times more affected. In the present analysis, leukemia, retinoblastoma and CNS tumors were common childhood malignancies. Furthermore, in the sub group of $<5$ year's retinoblastoma was commonest and, in the 5-9 and 10-14 years group leukemia was commonest. The histological sub types of childhood cancers are diverse and heterogenous, but in the present analysis ALL was the commonest histological sub type. Moreover, the common type of CNS tumors in children was astrocytoma in our population. A large multi centre study has also shown astrocytoma as most common brain tumors in children in the Indian population (Jain et al., 2011).

In this analysis, vast majority of patients with childhood cancers received CT as combination therapy with RT and /or surgery and also, as single modality in $41.4 \%$, followed by surgery+RT in $15.2 \%$ and $\mathrm{CT}+\mathrm{RT}$ in $13.8 \%$. CT was the major modality of treatment given, followed by radiotherapy and surgery in childhood cancers from Kolkata, India (Datta et al., 2010). Abandonment of treatment is the major cause of treatment failure in most studies. The proportion of patients abandoning treatment in developing countries has been reported to be around 2550\% (Arora et al., 2007; Mostert et al., 2012). In our study, the abandonment of treatment as defined earlier was seen in $5.5 \%$ of patients and $11.7 \%$ of parents refused treatment at the outset. There are various reasons for abandonment of complete treatment in different studies, most common being financial constraints and lack of parental education (Meremikwu et al., 2005; Arora et al., 2007; Slone et al., 2014). However, in our population the main issue will be to address the reasons for refusing start of treatment. Furthermore, our analysis has shown that median survival in children improves as the age group increases. This warrants further testing as the results were statistically not significant for comparison in the present analysis.

In conclusion, ascertaining the clinic-pathological profile of childhood cancers in our population is essential for allocation and management of resources for this small but important group of patients. So, a specialized child hood cancer registry has been advocated (Mehdiabadi et al., 2014). Furthermore, this type of analysis shed light on the pattern of care and necessitates the identification of socio-demographic determinants responsible for treatment compliance in childhood cancers.

\section{References}

Arora RS, Eden TO, Pizer B (2007). The problem of treatment abandonment in children from developing countries with cancer. Pediatr Blood Cancer, 49, 941-6.

Arora RS, Eden TO, Kapoor G (2009). Epidemiology of childhood cancer in India. Indian J Cancer, 46, 264-73.

Datta K, Choudhuri M, Guha S, Biswas J (2010). Childhood cancer burden in part of eastern India-population based cancer registry data for Kolkata (1997-2004), Asian Pac J Cancer Prev, 11,1283-8

Ferlay J, Bray F, Pisani P, Parkin DM (2004). Globocan 2002: cancer incidence. mortality and prevalence worldwide. IARC cancerbase No. 5, version 2.0., Lyon: IARC Press.

Ferlay J, Shin HR, Bray F, et al (2010). Estimates of worldwide burden of cancer in 2008: Globocan 2008. Int J Cancer, 127, 2893-917

Jain A, Sharma MC, Suri V, et al (2011). Spectrum of pediatric brain tumors in India: A multi-institutional study. Neurol India, 59,208-11

Mehdiabadi GB, Habibi R, Shariftabrizi A, Vossough P(2014). Epidemiologic survey of infantile cancer in Iran based on the data of the largest pediatric cancer referral center (AliAsghar children hospital), 1996-2005. Asian Pac J Cancer Prev, 15, 1211-7.

Meremikwu MM, Ehiri JE, Nkanga DG, et al (2005). Socioeconomic constraints to effective management of Burkitt's lymphoma in south-eastern Nigeria. Trop Med Int Health, 10, 92-8.

Mostert S, Gunawan S, Wolters E, et al (2012). Socio-economic status plays important roles in childhood cancer treatment outcome in Indonesia. Asian Pac J Cancer Prev, 13, 6491-6.

National Cancer Registry Programme (2013). Three-year report of the population based cancer registries of India: 2009-2011. NCDIR- ICMR, Bangalore.

National Cancer Registry Programme (2013). Consolidated report of hospital based cancer registries: 2007- 2011. Cancers in childhood. NCDIR-ICMR, Bangalore.

Satyanarayana L, Asthana S, Labani P (2014). Childhood cancer incidence in india: a review of population-based cancer registries. Indian Pediatr, 51, 218-20.

Slone JS, Chunda-Liyoka CC, Perez M, et al (2014). Pediatric malignancies, treatment outcomes and abandonment of pediatric cancer treatment in zambia. PLoS One, 9, 89102.

Wiangnon S, Veerakul G, Nuchprayoon I, et al (2011). Childhood cancer incidence and survival 2003-2005, Thailand: Study from the Thai pediatric oncology group. Asian Pac J Cancer Prev, 12, 2215-20. 